\title{
A new non-invasive method for treating insulin-reaction: intranasal lyophylized glucagon*
}

\author{
G. Slama ${ }^{1}$, C. Alamowitch ${ }^{1}$, N. Desplanque ${ }^{1}$, M. Letanoux ${ }^{1}$ and P.Zirinis ${ }^{2}$ \\ Department of Diabetes, Hôtel-Dieu Hospital, Paris and \\ ${ }^{2}$ Organon Research Laboratory, Sérifontaine, France
}

Summary. The main therapeutic indication for glucagon is the treatment of hypoglycaemia in insulin overdosed Type 1 (insulin-dependent) diabetic patients. We have previously shown that an intranasal spray of $7.5 \mathrm{mg}$ glucagon with deoxycholic acid as surfactant was able to correct an i.v. insulin-induced hypoglycaemia in diabetic patients. However, bioavailability and stability needed to be improved before intranasal glucagon could be introduced into clinical practice. This has now been achieved with a freeze-dried mixture of glucagon (1 mg) and glycocholic acid (1 mg) as a surfactant. Kinetics and efficacy have been controlled by (1) comparing subcutaneous and intranasal glucagon in 12 healthy non-hypoglycaemic subjects; (2) testing intranasal glucagon in six Type 1 diabetic patients in whom hypoglycaemia was induced by an i.v. bolus of insulin and (3) comparing subcutaneous and intranasal glucagon in six Type 1 diabetic patients in whom hypoglycaemia was induced by adding extra subcutaneous regular insulin to their usual morning dosage. Our results show that $1 \mathrm{mg}$ of intranasal glucagon is as effective as $1 \mathrm{mg}$ of subcutaneous glucagon in terms of the rise in blood glucose. Differences in kinetics between the subcutaneous and the intranasal routes may be observed: intranasal glucagon initiates the blood glucose rise earlier than does the subcutaneous form but the effect of the latter is more sustained. Glycocholic acid appears to be a perfectly tolerated agent in acute conditions. The use of intranasal lyophylized glucagon, for the reversal of hypoglycaemia in Type 1 diabetes, seems to be a clinically relevant alternative to its parenteral equivalent and should now be ready to be introduced in the market.

Key words: Hypoglycaemia therapy, glucagon, nasal route, diabetes mellitus.
Severe hypoglycaemic attacks occur frequently. They may be experienced by up to $25 \%$ of insulin-treated diabetic patients each year [1]. When the subject is unconscious and unable to swallow, only parenteral glucagon (usually $1 \mathrm{mg}$ ) or i.v. glucose can quickly correct the situation. However, injecting glucagon requires that relatives or friends be specially trained and it also requires aseptic manoeuvres which are not always performed very efficiently [2]. On the other hand, i.v. glucose, though highly efficient, requires professional skill.

In a recent study [3], we showed that an intranasal spray of $7.5 \mathrm{mg}$ of glucagon in a solution with $1 \%$ deoxycholic acid was able to correct $i$. v. induced hypoglycaemia in Type 1 (insulin-dependent) diabetic patients. We concluded that before intranasal glucagon could be intro-

\footnotetext{
* Presented at the $25^{\text {ih }}$ Annual Meeting of the EASD, Lisbon, September 19-23, 1989
}

duced as a realistic alternative in clinical practice, several difficulties needed to be overcome. In particular, a better adjuvant was needed to improve bioavailability and reduce cost, and a method to ensure the stability of glucagon in solution form was required [3]. We have found that the use of glucagon as a freeze-dried, lyophylised powder, seems to solve these critical questions.

\section{Subjects and methods}

The two following preparations of glucagon were used: (1) a commercially available glucagon set for s.c. injection (Novo Industrie SA, Neuilly, France), and (2) a freeze-dried pellet containing a mixture of $1 \mathrm{mg}$ glucagon and $1 \mathrm{mg}$ glycocholic acid (GCA) (Sigma, $\mathrm{La}$ Verpillère, France). This preparation was manufactured by Organon, (Sérifontaine, France), according to an original technical formulation (Organon Laboratory, P.Zirinis, patent pending). The pellets used for the entire study were manufactured in one batch, kept 


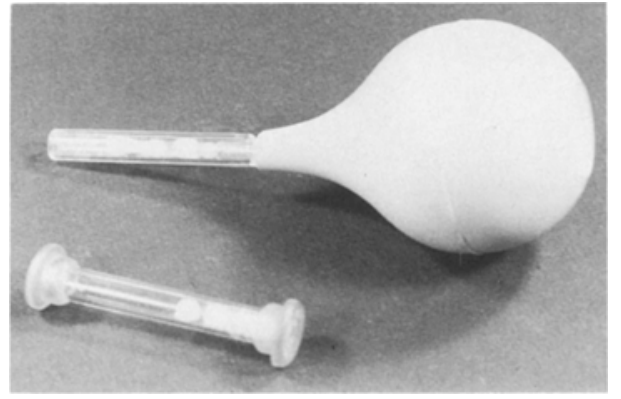

Fig.1. Picture of the unmounted, obturated glass tubing $(30 \mathrm{~mm}$ long) containing the lyophylised glucagon and a tubing mounted on the rubber bulb
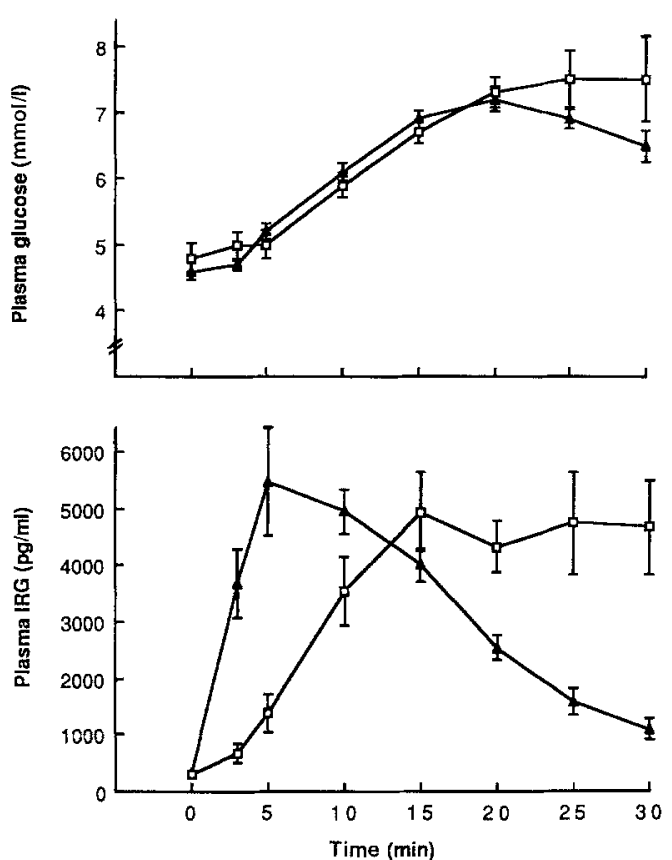

Fig. 2. Mean (SEM) plasma glucose and (immunoreactive glucagon) IRG levels in 12 normoglycaemic healthy subjects in response to $1 \mathrm{mg}$ glucagon given at $\mathrm{t}_{0}$ either s. c.: $(n=6 ; \square-\square)$, or IN as GCAglucagon: $(n=6 ; \boldsymbol{\wedge})$

in small glass tubes ( $30 \mathrm{~mm}$ long, $4 \mathrm{~mm}$ inner diameter) obturated at each end by rubber caps (Fig. 1), and stored at $+4{ }^{\circ} \mathrm{C}$. The preparation was kept in the refrigerator for at least 6 months before the study was started.

The pellet of glucagon was insufflated into one of the subject's nostrils via a rubber bulb (containing approximat $30 \mathrm{ml}$ air), which was cut at the extremity to fit the glass tubing. The powder was flushed from the tube by applying brisk pressure on the rubber bulb. The powder stuck lightly to the surface of the tubing, preventing it from being spilled, yet no residual powder was observable in the tubing system after insufflation.

The comparison between the two forms of glucagon was conducted in three consecutive sets of experiments: (1) in healthy, nonhypoglycaemic subjects, (2) in insulin-treated diabetic patients subjected to an i.v. induced, clinically light [1] hypoglycaemia, (3) in insulin-treated diabetic patients subjected to a more profound [1] s.c. insulin induced hypoglycaemic attack (a situation similar to that usually observed in clinical practice). After recovery, the patients were systematically asked (open-ended question) for possible side effects attributable to hypoglycaemia and/or glucagon administration.

The protocol was approved by the Hospital Ethical Committee and the informed subjects were volunteers.
Subcutaneous (s.c.) and intranasal (IN) glucagon

in non-hypoglycaemic subjects

Twelve healthy subjects aged 32.7 (2.8) years (mean \pm SEM) were randomly assigned to one of the two glucagon preparations: s. c. glucagon $(n=6)$ or intranasal GCA-glucagon $(n=6)$. They received the hormone at 08.30 hours after a $12 \mathrm{~h}$ fast, starting at an initial normal plasma glucose value of $4.8(0.22)$, and $4.8(0.11) \mathrm{mmol} / \mathrm{l}$ respectively $(p>0.05 ; \mathrm{NS})$.

\section{Intranasal glucagon for $i v$. induced (clinically light) hypoglycaemia in diabetic patients}

Six patients with Type 1, C peptide negative diabetes, aged 33.5 (3.6) years, were investigated after an overnight fast. Their last intermediate insulin injection was given $12 \mathrm{~h}$ before testing. We obtained a decrease in blood glucose value, below $3.3 \mathrm{mmol} / 1$ (assessed on Glucostix strips, Ames-Miles, Epernon, France), by injecting an i.v. bolus of regular insulin (U 40, Organon) at a dosage of $0.2 \mathrm{U} / \mathrm{kg}$ body weight, according to a protocol which has previously been described [3]. The actual mean plasma glucose value at which $1 \mathrm{mg} \mathrm{GCA-glu-}$ cagon was intranasally administered was $2.9(0.33) \mathrm{mmol} / \mathrm{l}$.

\section{Subcutaneous and intranasal glucagon for s.c. induced (more severe) hypoglycaemia}

Another group of six Type 1, C peptide negative diabetic subjects, aged $29.6(7.7)$ years, was subjected to a more severe s.c. insulin induced hypoglycaemia. All were routinely treated with two injections of a mixture of regular and NPH insulin. In order to obtain a hypoglycaemic episode the regular insulin component of the mixture injected in the morning, was increased by $50 \%$. The injection was given between 08.00 and 08.30 hours. We then waited for an overt insulin reaction. At this point, the patients were unable to react by themselves and take any carbohydrates. This was observed between 09.45 and 11.30 hours and corresponded to a concomittant capillary blood glucose value below $2.5 \mathrm{mmol} / 1$ (Glucostix readings using a Glucometer II, (Ames-Miles). The patients were then randomly assigned either to $1 \mathrm{mg}$ s.c. glucagon or to $1 \mathrm{mg}$ IN GCAglucagon. The plasma glucose values at which glucagon was administered were $2.3(0.16)$ and $2.1(0.23) \mathrm{mmol} / 1$ respectively $(p>0.05$; NS).

Plasma glucose was assayed using the glucose oxidase method (Beckman Glucose Analyser II, Fullerton, Calif., USA), and plasma immunoreactive glucagon (IRG) on radioimmunoassay (Oris Industries SA, Gif-sur-Yvette, France) (intra assay reproducibility: $4.4-4.9 \%$ ). All of the samples were assayed in the same batch.

\section{Statistical analysis}

Statistical analysis was carried out using the Student's $t$-test for paired data and multivariate (MANOVA) analysis of variance using SPSS-PC software. Results are given as mean ( \pm SEM).

\section{Results}

Figure 2 shows the mean plasma glucose and IRG concentrations recorded in the healthy subjects. No statistical difference could be found concerning peak values, values at $30 \mathrm{~min}$ or areas under the plasma glucose curves (Manova; NS). Concerning the concomittant plasma glucagon levels, the basal values were comparable: 320 (13) and 338 

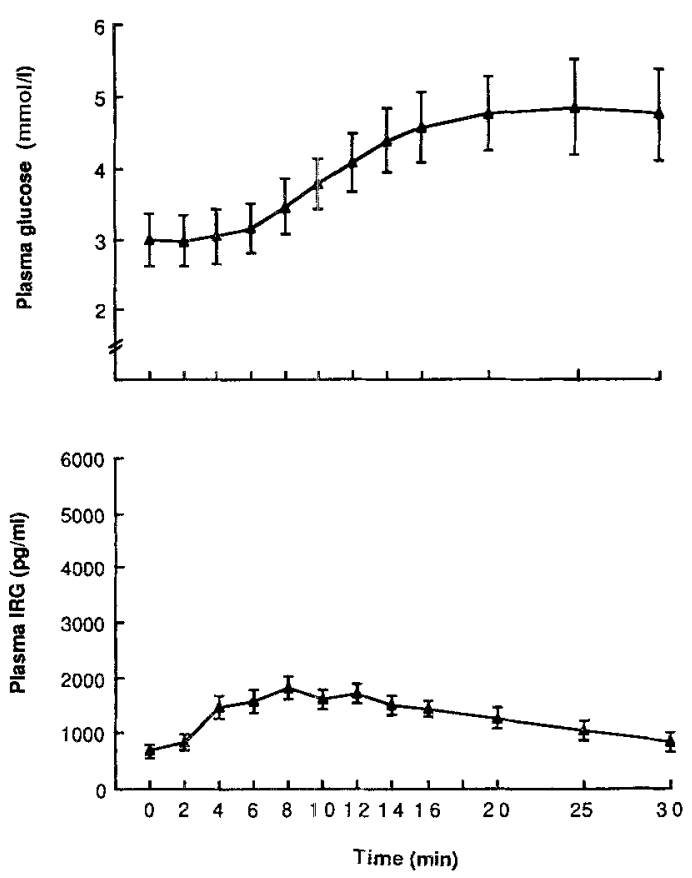

Fig. 3. Mean (SEM) plasma glucose and IRG levels in six insulin-dependent diabetic subjects submitted to i.v. induced hypoglycaemia and in response to $1 \mathrm{mg}$ intranasal GCA-glucagon ( $\Delta$ ) administered at $\mathrm{t}_{0}$
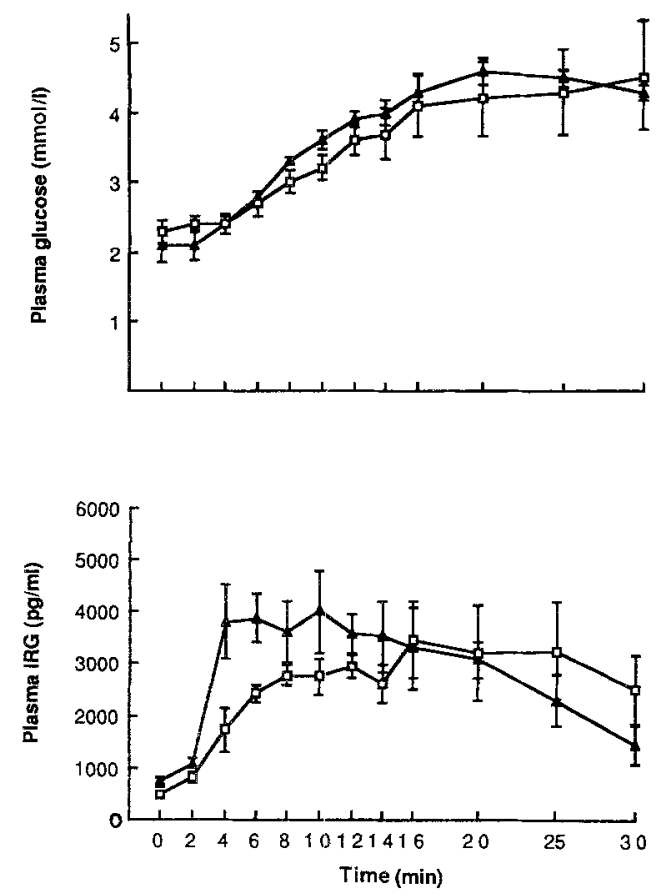

Fig. 4. Mean (SEM) plasma glucose and IRG levels in six insulin-dependent diabetic subjects submitted to s.c. insulin induced hypoglycaemia and in response to $1 \mathrm{mg}$ glucagon either s. c. $(n=3 ;[--\square)$ or $\mathrm{IN}$ (GCA-glucagon, $n=3 ; \mathbf{\Lambda}$ ) administered at $\mathrm{t}_{0}$

(25) $\mathrm{pg} / \mathrm{ml} ;(p>0.05 ; \mathrm{NS})$ but peak values $4933(690) ; 5468$ (950) and areas under the curves were significantly different (Manova: $p<0.001$ ), GCA-glucagon acted faster and higher than s.c. glucagon. However, at $30 \mathrm{~min}$, plasma IRG levels were almost still at their maximal values with s. c. glucagon, whereas with IN glucagon the levels had already returned to baseline.
Figure 3 shows the plasma glucose and IRG levels observed in Type 1 diabetic subjects submitted to an insulininduced light hypoglycaemia. After IN glucagon, plasma glucose levels increased steadily for $25 \mathrm{~min}$, attaining at that point a mean of $4.4(0.6) \mathrm{mmol} / \mathrm{l}$. The IRG plasma levels increased significantly with a mean peak value of 1822 (202) $\mathrm{pg} / \mathrm{ml}$ and then returned to baseline within $30 \mathrm{~min}$.

Figure 4 shows the plasma glucose and IRG levels recorded in diabetic patients in whom a more severe hypoglycaemia was obtained. No significant difference was observed either with plasma glucose or with IRG level kinetics.

\section{Discussion}

This study clearly demonstrates that $1 \mathrm{mg} \mathrm{IN}$ glucagon is at least as effective as $1 \mathrm{mg}$ s. c. glucagon in terms of blood glucose restoration after an insulin-induced hypoglycaemia.

Our results in healthy non-hypoglycaemic subjects confirm the findings of Pontiroli et al. $[4,5]$ and ourselves [3], that IN glucagon is rapidly reabsorbed through the nasal mucous membrane and promptly raises plasma IRG and glucose levels. Comparing $1 \mathrm{mg}$ glucagon administered intranasally and intramuscularly (i.m.) in healthy subjects, Pontiroli et al. concluded that the IN glucagon route seemed inferior to the i.m. route in terms of "blood glucose raising capacity; the ratio seems to be $1: 2$ " [4]. In the present study, $1 \mathrm{mg}$ glucagon IN with GCA seems to be as potent as $1 \mathrm{mg}$ s.c. glucagon in terms of plasma glucose and maximal plasma IRG levels; the kinetics are more alert via the IN route but have a more sustained effect with s. c. delivery.

The results observed with $1 \mathrm{mg}$ GCA-glucagon in insulin-dependent diabetic subjects submitted to a relatively light i. v. insulin-induced hypoglycaemia are totally superposable, in terms of plasma glucose, with those obtained with $7.5 \mathrm{mg}$ glucagon IN as a spray with deoxycholic acid as surfactant. It has been argued however, that in the absence of a control group, one cannot be sure that the phenomenon observed is not simply due to a spontaneous blood glucose recovery [6], since the effect of an i.v. insulin bolus is very short-lived. As indicated in the literature, and from the largely elevated plasma IRG values that can be physiologically observed, or in response to insulin-induced hypoglycaemia, many arguments can be posited against this hypothesis. It should be noted however, that $1 \mathrm{mg}$ IN glucagon is much higher than the minimum effective dosage and maximum plasma glucose levels were observed whether peak IRG levels were 5468 (Fig. 2), 4000 (Fig.4) or 1822 (Fig. 3) pg/ml. We also have to consider our ethical inability to obtain a control group of diabetic subjects submitted to hypoglycaemia and left without treatment (placebo group) [7]. To overcome this bias, we compared the IN route to the long established $1 \mathrm{mg}$ s.c. route of glucagon administration [8] in Type 1 diabetic patients experiencing severe hypoglycaemia after an overdose of s.c. administered insulin. We decided to increase, by $50 \%$, the regular insulin component of the total morning dosage. The observed plasma glucose nadir 
levels were quite low $(<2.5 \mathrm{mmol} / \mathrm{l})$ in all of the subjects tested. Similarly, the hypoglycaemic state was corrected within 10 min and the plasma IRG levels were not statistically different between the two forms of glucagon administration. The number of subjects in this third group was indeed too small to allow definitive conclusions to be drawn. However, we have since conducted a clinical experience with young diabetic subjects staying in summer camps who were managed by a non-medical staff (ongoing experiment not yet published).

We observed minor side effects with the surfactant used in the present study due to the IN route: tingling sensations in the nose for 1 or $2 \mathrm{~min}$; with GCA-glucagon appeared to be better tolerated than deoxycholic acid used in our previous experiment. We did not visually or histologically study the nasal mucous membrane, because no chronic effects were expected in this drug which is only administered in acute situations.

The side effects observed with s.c. glucagon (gastrointestinal discomfort, nausea) were observed in six of the healthy and two of the diabetic subjects. No such effects were observed with the IN route. This may be explained by a more fleeting increase in IRG levels. This is especially important for children who often experience severe nausea and vomitting after s. c. glucagon.

In addition, we have the distinct impression, from our observations and from the patients' opinions, that IN glucagon acts more quickly than s. c. injection, but we are unable, under the conditions of our protocol, to quantify this. These observations are also concordant with those of Pontiroli et al. [5, 9].

We conclude that $1 \mathrm{mg}$ IN lyophylised glucagon is at least as efficient as $1 \mathrm{mg}$ s.c. glucagon in correcting insulin-induced hypoglycaemia in diabetic patients in a situation similar to that observed in clinical practice. The flatter IRG curves observed with diabetic patients as compared to non-diabetic subjects might be due to the extensive variations in the response among the subjects, but may also be due to a better dispersion of the powder on the nasal mucosa after an additional voluntary inspiration.
Acknowledgements. We are grateful to Mr. M. Mesbah (Unité U 21, INSERM) for his help in the statistical evaluation of our results and to Mme K. Bean for revising the manuscript. This work was supported by a grant from Pierre et Marie Curie University (Paris VI), France.

\section{References}

1. Goldgewicht C, Slama G, Papoz L, Tchobroutsky G (1983) Hypoglycaemic reactions in 172 Type 1 (insulin-dependent) diabetic patients. Diabetologia 24: 95-99

2. Mühlhauser I, Berger M, Sonnenberg G, Koch J, Jörgens V, Schernthaner G, Scholz V (1985) Incidence and management of severe hypoglycemia in 434 adults with insulin-dependent diabetes mellitus. Diabetes Care 8: 268-273

3. Freychet L, Rizkalla SW, Desplanque N, Basdevant A, Zirinis P, Tchobroutsky G, Slama G (1988) Effect of glucagon on blood glucose levels in healthy subjects and hypoglycaemic patients with insulin-dependent diabetes. Lancet I: 1364-1366

4. Pontiroli AE, Alberetto M, Pozza G (1983) Intranasal glucagon raises blood glucose concentrations in healthy volunteers. Br Med J 287: 462-463

5. Pontiroli AE, Alberetto M, Pozza G (1985) Metabolic effects of intranasally administered glucagon: comparison with intramuscular and intravenous injection. Acta Diab Lat 22: 103-109

6. Arnold JD, Swift GF (1988) Intranasal glucagon for hypoglycaemia. Lancet II: 216

7. Slama G, Freychet L, Desplanque N (1988) Intranasal glucagon for hypoglycaemia. Lancet II: 799

8. Elrick H, Witten TA, Yoshiaki A (1958) Glucagon treatment of insulin reactions. N Engl J Med 258: 476-480

9. Alberetto M, Pontiroli AE, Calderara A, Pajetta E, Pozza G (1988) Intranasal glucagon for hypoglycaemic crises in patients with Type 1 (insulin-dependent) diabetes. Diabetologia 31: $463 \mathrm{~A}$

Received: 5 February 1990

and in revised form: 28 May 1990

Dr. G. Slama

Department of Diabetes

Hôtel-Dieu Hospital

1, Place du Parvis Notre-Dame

F-75004 Paris

France 